\title{
Amorphous Gallium Oxide Thin Film Grown by Atomic Layer Deposition for High-Performance and Flexible Deep-Ultraviolet Photodetector
}

\author{
Kang Min Lee, Se Eun Kim, Hyeok Jae Lee, Sang Woon Lee \\ Department of Energy Systems Research and Department of Physics, Ajou University \\ Suwon 16499, Republic of Korea \\ leekigg@ajou.ac.kr; kimss3381@naver.com; gurwoqod154@ajou.ac.kr; slee01@ajou.ac.kr
}

\section{Extended Abstract}

Deep-ultraviolet (DUV) light with a wavelength $(\lambda)$ of 200 300 nm has been spotlighted for the photolysis, water or air purification. Interestingly, DUV light does not exist on the earth surface naturally, because DUV coming from the sun is absorbed by ozone in the atmosphere.[1] Thus, DUV can be used for communication or missile tracking thanks to a weak background interference. For those applications, a development of high-performance DUV photodetector is necessary.[2] DUV detection is also important for sensing biological molecules because some of biological molecules absorption and florescence spectrum is within the range of DUV wavelength.[3] In the meantime, DUV light is dangerous and harmful to human body because of its high energy $(>4 \mathrm{eV})$. Therefore, the development of high-performance DUV photodetector is inevitable to operate the DUV light safely. For the DUV detection, absorber with wide bandgap $(>4.5 \mathrm{eV})$ is required such as AlGaN. Unfortunately, most of existing DUV photodetectors exhibited a poor performance in the DUV detection despite the use of the epitaxial AlGaN layer.[4] In addition, the growth of epitaxial AlGaN layer must be done at high temperature $\left(>1000{ }^{\circ} \mathrm{C}\right.$ ) using complex buffer layers. $\mathrm{Ga}_{2} \mathrm{O}_{3}$ is a good candidate for the DUV detection because of its wide bandgap ( 5 $\mathrm{eV}$ ), however, photodetectors using epitaxial $\mathrm{Ga}_{2} \mathrm{O}_{3}$ films exhibited a slow response speed with low responsivity.[5]

Here, we demonstrated a high-performance DUV photodetector using an amorphous gallium oxide thin films $\left(\mathrm{GaO}_{\mathrm{x}}\right)$ grown by atomic layer deposition (ALD) at a low growth temperature of $<250{ }^{\circ} \mathrm{C}$ for the first time. Interestingly, the amorphous $\mathrm{GaO}_{\mathrm{x}}$ showed a wide bandgap of $(\sim 4.9 \mathrm{eV})$ which is comparable with epitaxial $\mathrm{Ga}_{2} \mathrm{O}_{3}$ films. The photodetector using 30-nm-thick amorphous $\mathrm{GaO}_{\mathrm{x}}$ film showed a fast response (as short as $\sim 3$ us) with high responsivity $(\sim 45 \mathrm{~A} / \mathrm{W}$ at $\lambda=$ $253 \mathrm{~nm}$ ) which outperforms conventional DUV photodetectors. The cut-off wavelength is $\sim 300 \mathrm{~nm}$ that does not respond to visible lights, and the photodetector detects only DUV wavelengths selectively. It should be noted that general substrates such as a glass and quartz can be used for the DUV photodetector owing to the amorphous phase of $\mathrm{GaO}_{\mathrm{x}}$ film, which enables a practical application of the fabrication protocol. Finally, we demonstrated a flexible DUV photodetector fabricated on polyimide substrate which showed a reliable detection of DUV with the repetitive bending cycles beyond $>3000$ times at a bending radius of $<10 \mathrm{~mm}$. Interestingly, the performance was maintained under the bending radius of $<2 \mathrm{~mm}$ by a stress engineering. This process scheme will provide an economically useful solution for the development of DUV sensor for various applications.

\section{References}

[1] C. S. Cockell, "Biological effects of high ultraviolet radiation on early Earth-a theoretical evaluation," Journal of theoretical biology, vol. 193, pp. 717-729, 1998.

[2] Q. Zhang, J. Jie, S. Diao, Z. Shao, Q. Zhang, L. Wang, W. Deng, W. Hu, H. Xia, X. Yuan, S.-T. Lee., "Solutionprocessed graphene quantum dot deep-UV photodetectors," ACS nano, vol. 9, pp. 1561-1570, 2015.

[3] C. E. Lovelock and S. A. Robinson, "Surface reflectance properties of Antarctic moss and their relationship to plant species, pigment composition and photosynthetic function," Plant, Cell \& Environment, vol. 25, pp. 1239-1250, 2002.

[4] P. Sandvik, K. Mi, F. Shahedipour, R. McClintock, A. Yasan, P. Kung, M. Razeghi, "AlxGaa ${ }_{1-x} N$ for solar-blind UV detectors," Journal of crystal growth, vol. 231, pp. 366-370, 2001. 
[5] D. Guo, Z. Wu, P. Li, Y. An, H. Liu, X. Guo, H. Yan, G. Wang, C. Sun, L. Li, and W. Tang, "Fabrication of $\beta-\mathrm{Ga}_{2} \mathrm{O}_{3}$ thin films and solar-blind photodetectors by laser MBE technology," Optical Materials Express, vol. 4, pp. 1067-1076, 2014. 